\title{
Multiplicity, Kinematics, and Rotation Rates of Very Young Brown Dwarfs in Cha I
}

\author{
Viki Joergens, Ralph Neuhäuser \\ Max-Planck-Institut für Extraterrestrische Physik, Giessenbachstr. 1, \\ D-85748 Garching, Germany
}

Eike W. Guenther

Thüringer Landessternwarte Tautenburg, Sternwarte 5, D-07778

Tautenburg, Germany

Matilde Fernández

Instituto de Astrofísica de Andalucía (CSIC), Apdo. 3004, E-18080 Granada, Spain

\author{
Fernando Comerón \\ European Southern Observatory, Karl-Schwarzschild-Str. 2, D-85748 \\ Garching, Germany
}

\begin{abstract}
We have studied twelve very young (1-5 Myr) bona fide and candidate brown dwarfs in the Cha I star forming region in terms of their kinematic properties, the occurrence of multiple systems among them as well as their rotational characteristics. Based on high-resolution spectra taken with UVES at the VLT $(8.2 \mathrm{~m})$, radial and rotational velocities have been measured. A kinematic study of the sample showed that their radial velocity dispersion is relatively small suggesting that they are not ejected during their formation as proposed in recent formation scenarios. By means of time-resolved UVES spectra, a radial velocity survey for close companions to the targets was conducted. The radial velocities of the targets turned out to be rather constant setting upper limits for the mass $\mathrm{M}_{2} \sin i$ of possible companions to $0.1 \mathrm{M}_{\mathrm{Jup}}-2 \mathrm{M}_{\mathrm{Jup}}$. These findings hint at a rather low $(\leq 10 \%)$ multiplicity fraction of the studied brown dwarfs. Furthermore, a photometric monitoring campaign of the targets yielded the determination of rotational periods for three brown dwarf candidates in the range of 2.2 to 3.4 days. These are the first rotational periods for very young brown dwarfs and among the first for brown dwarfs at all.
\end{abstract}

\section{Introduction}

Although a large number of brown dwarfs have been detected up to date, fundamental parameters have been measured only for a small subset. Consequently, there are still a lot of open questions, for example, the mechanism that leads to the formation of brown dwarfs is still poorly constrained. 


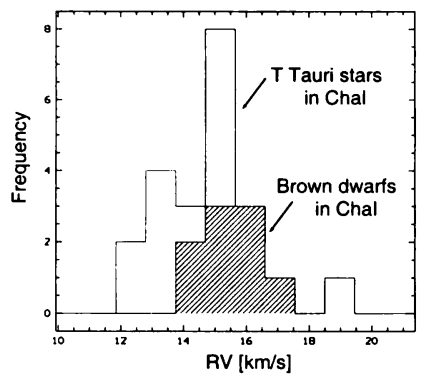

Figure 1. Radial velocity distribution for 9 bona fide and candidate brown dwarfs (hashed) and for $22 \mathrm{~T}$ Tauri stars in Cha I.

We have studied a population of twelve very young bona fide and candidate brown dwarfs in the ChaI star forming region, Cha H $\alpha 1$ to 12 (Comerón et al. 1999, 2000, Neuhäuser \& Comerón 1998, 1999) by means of high-resolution spectroscopy $(\lambda / \Delta \lambda=40000)$ with UVES at the $8.2 \mathrm{~m}$ Kueyen telescope of the VLT as well as by a photometric monitoring campaign carried out at the Danish $1.5 \mathrm{~m}$ telescope at ESO. These observations yielded radial and rotational velocities as well as rotational periods. The studied brown dwarfs are only a few million years old and their observation allows insights into the formation and early evolution of brown dwarfs.

\section{UVES spectra I: Kinematic study}

High-resolution spectroscopic observations were conducted with the echelle spectrograph UVES attached to the $8.2 \mathrm{~m}$ Kueyen telescope of the VLT. At least two spectra separated by a few weeks have been taken of nine of the twelve targets. The wavelength regime from $6600 \AA$ to $10400 \AA$ was covered with a spectral resolution of $\lambda / \Delta \lambda=40000$. Radial velocities have been measured by a crosscorrelation of plenty of stellar lines of the object spectra against a template spectrum. Telluric lines have been used as wavelength reference. The achieved precision of the relative radial velocities range between $80 \mathrm{~m} \mathrm{~s}^{-1}$ and $600 \mathrm{~m} \mathrm{~s}^{-1}$, depending on the $\mathrm{S} / \mathrm{N}$ of the individual spectra.

Recently, it has been proposed, that brown dwarfs are ejected protostars which have been cut off from the gas reservoir in the early accretion phase of the star formation process and could have therefore not accreted to stellar masses (Reipurth \& Clarke 2001). A kinematic study of the sample (Joergens \& Guenther 2001) showed that their radial velocity dispersion is relatively small $\left(2.2 \mathrm{~km} \mathrm{~s}^{-1}\right)$. It is significantly smaller than the radial velocity dispersion of the $\mathrm{T}$ Tauri stars in the field $\left(3.6 \mathrm{~km} \mathrm{~s}^{-1}\right)$ and slightly larger than that one of the surrounding molecular gas $\left(1.2 \mathrm{~km} \mathrm{~s}^{-1}\right)$. The distributions are displayed in Fig. 1. These results give suggestive evidence that there is no run-away brown dwarf among the studied sample. It cannot be ruled out that some of them have a larger 3D velocity dispersion. However, the studied brown dwarfs occupy a field of less than $12^{\prime} \times 12^{\prime}$ at a distance of $160 \mathrm{pc}$. Brown dwarfs born within this field and ejected during the early accretion phase in directions with a significant 

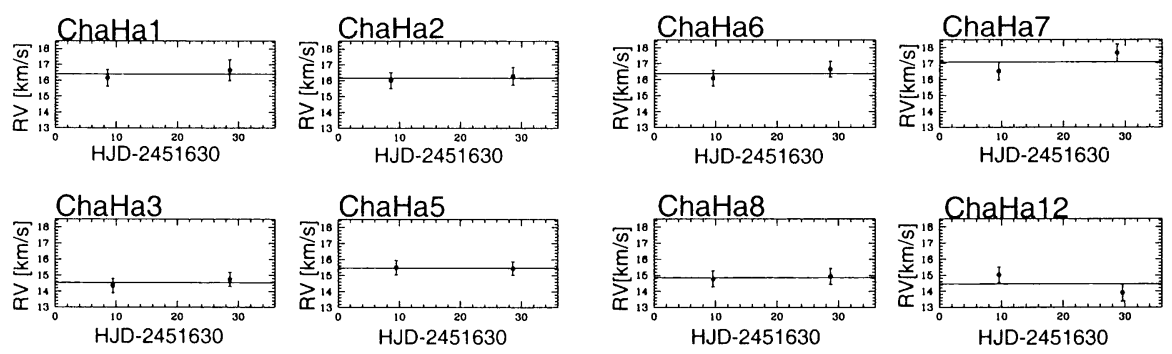

Figure 2. Radial velocity vs. time.

fraction perpendicular to the line-of-sight, would have flown out of the field a long time ago, given the age of the objects of $1-5 \mathrm{Myr}$. This is true even for the smallest ejection velocities of $2 \mathrm{~km} \mathrm{~s}^{-1}$ calculated by theorists (see also next paragraph). Therefore, the measurement of radial velocities is the very method to test if objects born in the field have significantly high velocities due to dynamical interactions during their formation process.

Very recent dynamical calculations (Bate et al. 2002, Sterzik \& Durisen this volume) hint at rather small ejection velocities suggesting the possibility that the imprint of the ejection in the kinematics might not be an observable effect. They predict a velocity dispersion of ejected brown dwarfs of $2 \mathrm{~km} \mathrm{~s}^{-1}$ in $3 \mathrm{D}$ and $1.2 \mathrm{~km} \mathrm{~s}^{-1}$ in $1 \mathrm{D}$. On the other hand, they predict that $10 \%$ of the brown dwarfs have a larger velocity than $5 \mathrm{~km} \mathrm{~s}^{-1}$ due to dynamical interactions. The velocities of the bona fide and candidate brown dwarfs in Cha I cover a total range of only $2.6 \mathrm{~km} \mathrm{~s}^{-1}$. Therefore, it is concluded that the ejection-model for the formation of brown dwarfs is not a likely formation mechanism for the brown dwarfs in Cha I.

\section{UVES spectra II: RV Survey for companions}

By means of time-resolved UVES spectra, a radial velocity survey for close companions to the targets was conducted. For each of the objects Cha $\mathrm{H} \alpha 1,2$, $3,5,6,7,8$ and 12 spectra have been taken in two nights separated by a few weeks. For Cha $\mathrm{H} \alpha 4$ spectra have been taken in 11 nights, separated by weeks to years.

We have found that the radial velocities for Cha H $\alpha 1,2,3,5,6,7,8$ and 12 are constant within the measurements uncertainties, whereas Cha $\mathrm{H} \alpha 4$ shows small but significant variations (Fig. 2). Details will be published in a forthcoming paper (Joergens et al., in prep.).

An upper limit for the semiamplitude of a variation caused by a hypothetical companion was determined for each object by assuming that the total variability amplitude was recorded. Subsequently, upper mass limits for the hypothetical companions have been estimated. For this calculation an orbital separation of $0.1 \mathrm{AU}$ was assumed. Primary masses from Comerón et al. (1999, 2000) have been used. Furthermore, circular orbits and an inclination of $90^{\circ}$ were assumed. The assumption of $i=90^{\circ}$ means that the derived mass limits for companions are upper limits for the minimum masses $\mathrm{M}_{2} \sin i$. 


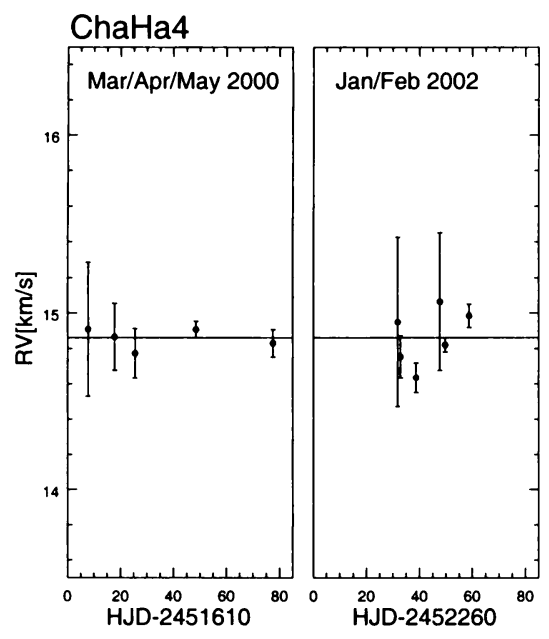

Figure 3. Radial velocity vs. time.

The upper limits for the semiamplitude of the 'constant' objects (Fig. 2, left panel) range between $30 \mathrm{~m} \mathrm{~s}^{-1}$ for Cha $\mathrm{H} \alpha 5$ and $600 \mathrm{~m} \mathrm{~s}^{-1}$ for Cha $\mathrm{H} \alpha 7$ and 12. The deduced upper limits for $\mathrm{M}_{2} \sin i$ range between a tenth of a Jupiter mass (Cha $\mathrm{H} \alpha 5)$ and 1-2 Jupiter masses (Cha H $\alpha$ and 12). The small but significant variations of the radial velocity of $\mathrm{Cha} \mathrm{H} \alpha$, if caused by a companion, correspond to a companion mass $\mathrm{M}_{2} \sin i$ of $0.8 \mathrm{M}_{\mathrm{Jup}}$.

In summary, no evidence for substellar companions around the targets has been found, aside from variations of the radial velocity of $\mathrm{ChaH} \alpha 4$, which could be due to a companion of less than $1 \mathrm{M}_{\mathrm{Jup}}$. There is the possibility that there are planetary mass companions with masses below one Jupiter mass since the presented observations were not sensitive enough to detect those. Furthermore, spectroscopic binaries in the sample might not have been detected due to the non-observation at the critical orbital phases. However, the overall picture that arises is that the multiplicity fraction of the studied brown dwarfs is rather low $(\leq 10 \%)$.

\section{Photometric monitoring: Rotational periods}

A photometric monitoring campaign has been carried out at the Danish $1.5 \mathrm{~m}$ telescope at ESO in order to study the time dependence of the brightness of the objects. Rotational periods have been measured by tracing modulations of the light curves due to magnetically induced surface spots. Rotational periods have been determined for the three brown dwarf candidates $\mathrm{Cha} \mathrm{H} \alpha 2$, Cha $\mathrm{H} \alpha 3$ and Cha $\mathrm{H} \alpha 6$ of $2.8 \mathrm{~d}, 2.2 \mathrm{~d}$ and $3.4 \mathrm{~d}$, respectively (Joergens et al. 2002a,b). See Fig. 3 and 4 for phase-folded light curves. The periods are consistent with measurements of rotational velocities $v \sin i$ from UVES spectra for the objects $\left(8-26 \mathrm{~km} \mathrm{~s}^{-1}\right)$. The observations show that brown dwarfs at an age of $1-5 \mathrm{Myr}$ display surface spots like $\mathrm{T}$ Tauri stars and are moderately fast rotators in contrast to rapidly rotating old brown dwarfs consistent with them being in 

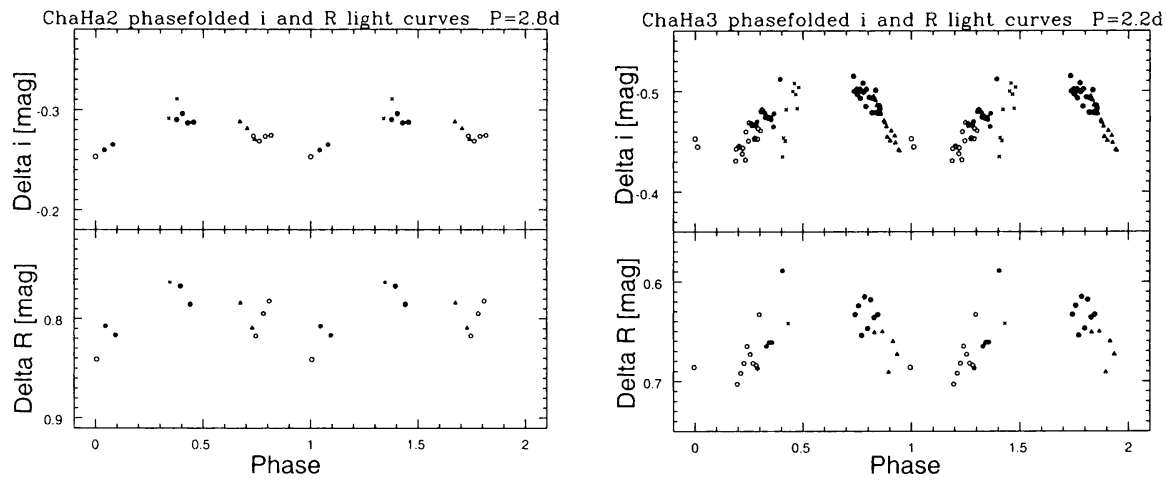

Figure 4. Phase-folded $\mathrm{i}$ and $\mathrm{R}$ band light curves of Cha $\mathrm{H} \alpha 2$ and Cha H $\alpha 3$. Different symbols denote data points obtained in different nights.
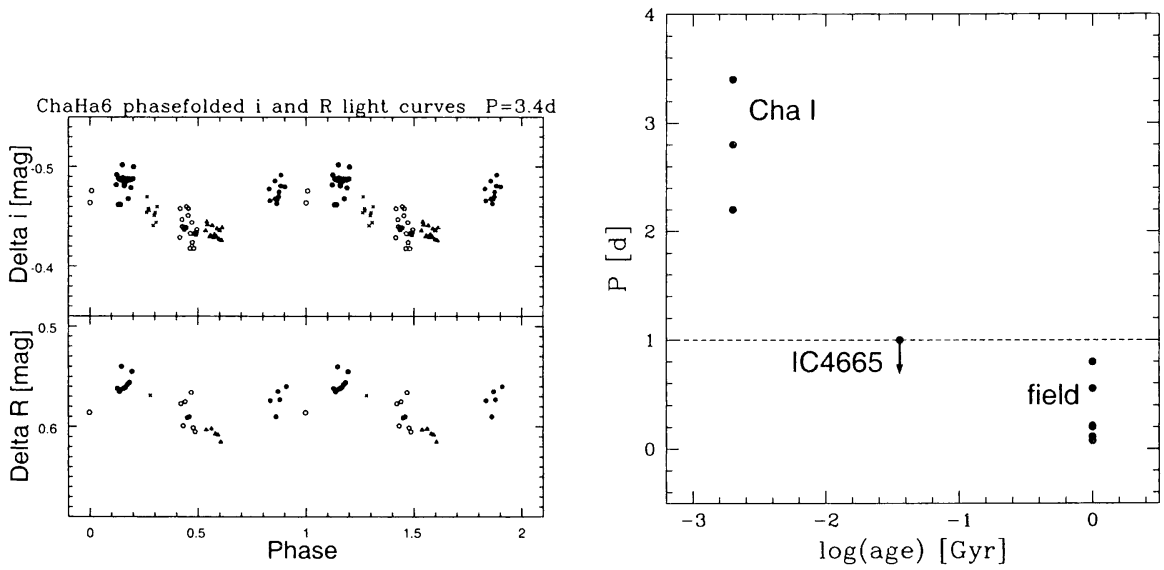

Figure 5. Left: Phase-folded light curve of Cha $\mathrm{H} \alpha 6$. Right: Evolution of angular momentum from $1 \mathrm{Myr}$ to $1 \mathrm{Gyr}$. Rotational period $\mathrm{P}$ vs. age for $\mathrm{Cha \textrm {H }} \alpha 2,3$ and 6 , brown dwarfs in the cluster IC4665 (Eislöffel \& Scholz 2001) and brown dwarfs in the field (Bailer-Jones \& Mundt 2001, Martín et al. 2001, Clarke et al. 2002). 
an early contracting stage. A comparison with rotational periods at $36 \mathrm{Myr}$ (Eislöffel \& Scholz 2001) indicates that most of the acceleration of brown dwarfs takes place in the first 30 million years or less of their lifetime (cf. Fig. 4, right panel).

\section{Discussion and conclusions}

The kinematic study of brown dwarfs in Cha I based on the measurement of precise radial velocities from high-resolution UVES spectra showed that their radial velocities have a dispersion of only $2.2 \mathrm{~km} \mathrm{~s}^{-1}$ and span a total range of only $2.6 \mathrm{~km} \mathrm{~s}^{-1}$. We therefore conclude that the ejection-model for the formation of brown dwarfs (Reipurth \& Clarke 2001) is not a likely formation mechanism for the studied brown dwarfs since first calculations (Bate et al. 2002, Sterzik \& Durisen 2002, this volume) predict that $10 \%$ of the brown dwarfs should have a larger velocity than $5 \mathrm{~km} \mathrm{~s}^{-1}$ due to the ejection.

The small binary fraction $(\leq 10 \%)$ found in the presented RV survey is in agreement with the result of a direct imaging survey for wide, low-mass companions to the same objects (Neuhäuser et al. (2002), Joergens et al. (2001), Neuhäuser et al. this volume). Combining both surveys, a wide range of possible companion separations has been covered. The exact separations depend on the companion masses. For example, for brown dwarf companions $\left(<13 \mathrm{M}_{\text {Jup }}\right)$ to the targets, separations $<3 \mathrm{AU}$ and between 50 and $1000 \mathrm{AU}$ were covered. With more restricted separations $(<0.1 \mathrm{AU}$ and $300-1000 \mathrm{AU})$ the surveys were sensitive also to companion masses down to $1 \mathrm{M}_{\text {Jup }}$.

These results seem to be in contrast with the high multiplicity fraction observed for very young stars and may suggest that brown dwarfs form not by direct collapse of unstable cloud cores as stars. However, a significant comparison of the multiplicity fraction of stars and brown dwarfs at very young ages is still hampered by small-number statistics in the substellar regime. Such a study has to compare multiplicity fractions in a certain separation range, which has to agree for both samples. Furthermore, it is possible that the gravitational collapse of cloud cores of brown dwarf masses does not yield as much multiple systems as the collapse of clouds of solar masses. In addition, stars can have companions, which have only a tenth of the mass of the primary, whereas a companion of a brown dwarf with such a low mass ratio would be already a planet. However, there is still a lot to do for observers as well as for theorists in order to understand in which way objects of brown dwarf masses are formed.

Finally, we have determined rotational periods for the three brown dwarf candidates Cha $\mathrm{H} \alpha 2$, Cha $\mathrm{H} \alpha 3$ and $\mathrm{ChaH} \alpha 6$ of 2.2 to 3.4 days by means of a photometric monitoring campaign. These are the first rotational periods for very young brown dwarfs and among the first for brown dwarfs at all. They provide valuable data points in an as yet almost unexplored region of the substellar period-age diagram. A comparison of the determined rotational periods at the age of a few million years with rotational properties of older brown dwarfs ( $>36$ Myr, Eislöffel \& Scholz 2001) shows that most of the acceleration of brown dwarfs during the contraction phase takes place within the first $30 \mathrm{Myr}$ or less. It is known that Cha $\mathrm{H} \alpha 2$ and 6 have optically thick disks (Comerón et al. 2000 ), therefore magnetic braking due to interactions with the disk may play a 
role for them. This is suggested by the fact, that among the three brown dwarf candidates with determined periods, the one without a detected disk, $\mathrm{Cha} \mathrm{H} \alpha 3$, has the shortest period. If the interaction with the disk is responsible for the braking, the results from Eislöffel \& Scholz (2001) indicate that brown dwarf inner disks have been dissipated at an age of $36 \mathrm{Myr}$. These limits for the time scale of disk dissipation for brown dwarfs are very similar to those for $\mathrm{T}$ Tauri stars, which are known to dissolve their inner disks within about the first $10 \mathrm{Myr}$ (e.g. Calvet et al. 2000).

Further measurements of rotational periods for brown dwarfs are muchneeded in order to complete the picture of angular momentum evolution in the substellar regime as well as that of rotationally induced phenomena, like dynamo activity and meteorological processes.

\section{References}

Bailer-Jones C.A.L. \& Mundt R. 2001, A\&A 367, 218

Bate M.R., Bonnell I.A. \& Bromm V. 2002, MNRAS 332, L65

Calvet N., Hartmann L. \& Strom S.E. 2000, 'Evolution of Disk Accretion', In: Protostars and Planets IV, ed. by Mannings V., Boss A.P., Russell S.S., Univ. of Arizona Press, Tucson, p. 377

Clarke F.J., Tinney C.G. \& Covey K.R. 2002, MNRAS 332, 361

Comerón F., Rieke G.H. \& Neuhäuser R. 1999, A\&A 343, 477

Comerón F., Neuhäuser R. \& Kaas A.A. 2000, A\&A 359, 269

Eislöffel J. \& Scholz A. 2001, 'Rotation and Atmospheres of Brown Dwarfs'. In: Origins of stars and planets: The VLT view, ESO workshop, Garching, Germany, April 24-27, 2001, in press

Joergens V., Guenther E., Neuhäuser R. et al. 2001 'Multiplicity of young brown dwarfs in Cha I'. In: Origins of stars and planets: The VLT view, ESO workshop, Garching, Germany, April 24-27, 2001, in press, astro$\mathrm{ph} / 0106185$

Joergens V. \& Guenther E.W. 2001, A\&A 379, L9

Joergens V., Fernandéz M., Neuhäuser R. \& Guenther E.W. 2002a, Poster proceeding of the Star Spot Thinkshop, held in Potsdam, Germany in May 2002 , in press, astro-ph/0206137

Joergens V., Fernandéz M. \& Neuhäuser R. 2002b, A\&A subm.

Martín E.L., Zapatero Osorio M.R. \& Lehto H.J. 2001, ApJ 557, 822

Neuhäuser R. \& Comerón F. 1998, Science 282, 83

Neuhäuser R. \& Comerón F. 1999, A\&A 350, 612

Neuhäuser R. \& Brandner W., Alves J., Joergens V., Comerón F. 2002, A\&A 384,999

Reipurth B. \& Clarke C. 2001, ApJ 122, 432 


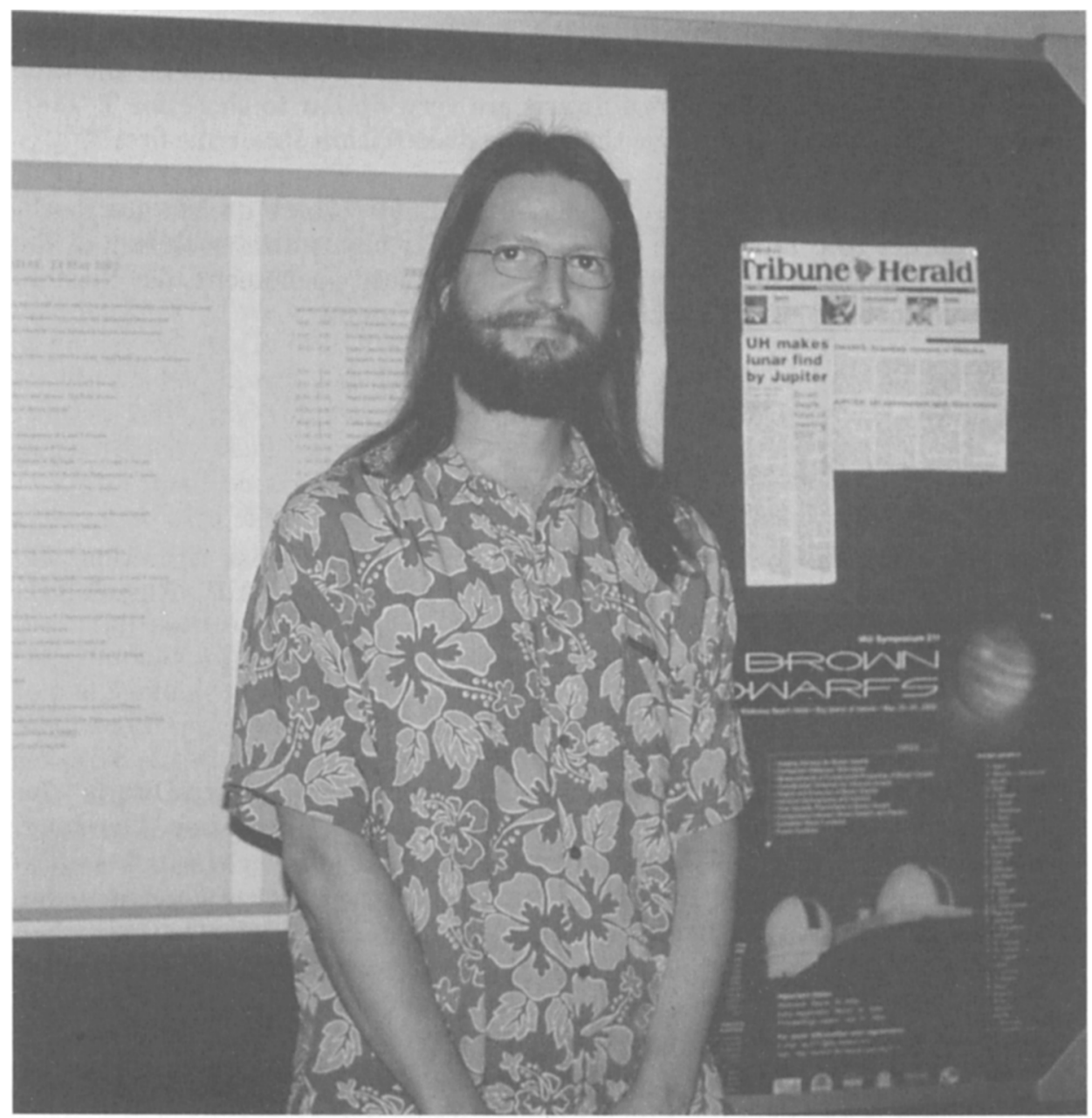

\section{Ralph Neuhäuser}

